\title{
Alterations of caveolin-1 expression in a mouse model of delayed cerebral vasospasm following subarachnoid hemorrhage
}

\author{
YE XIONG, XUE-MIN WANG, MING ZHONG, ZE-QUN LI, ZHI WANG, \\ ZUO-FU TIAN, KUANG ZHENG and XIAN-XI TAN
}

Department of Neurosurgery, First Affiliated Hospital of Wenzhou Medical College, Wenzhou, Zhejiang 325000, P.R. China

Received November 15, 2014; Accepted January 13, 2016

DOI: $10.3892 /$ etm.2016.3568

\begin{abstract}
The aim of the present study was to evaluate the expression levels of caveolin-1 in the basilar artery following delayed cerebral vasospasm (DCVS) in a rat model of subarachnoid hemorrhage (SAH), in order to investigate the association between caveolin-1 and DCVS, and its potential as a treatment for DCVS of SAH. A total of 150 Sprague Dawley rats were randomly allocated into blank, saline and SAH groups. The SAH and saline groups were subdivided into days 3, 5, 7 and 14 following the establishment of the model. The murine model of SAH was established by double injection of autologous arterial blood into the cisterna magana and DCVS was detected using Bederson neurological severity scores. Hematoxylin and eosin (HE) staining was used to observe the inner perimeter of the basilar artery pipe and variations in the thickness of the basilar artery wall. Alterations in the levels of caveolin-1 protein in the basilar artery were measured using immunofluorescence and western blot analysis; whereas alterations in the mRNA expression levels of caveolin-1 were detected by reverse transcription-quantitative polymerase chain reaction. In the present study, 15 mice succumbed to SAH-induced DCVS in the day $3(n=3), 5(n=5)$ and $7(n=2)$ groups. No mortality was observed in the blank control and saline groups during the process of observation in the SAH group, All mice in the SAH groups exhibited Bederson neurological severity scores $\geq 1$; whereas no neurological impairment was detected in the blank and normal saline groups, demonstrating the success of the model. HE staining was used to assess vasospasm and the results demonstrated that the inner perimeter of the basal artery pipe decreased at day 3 in the SAH group; whereas values peaked in the day 7 group. The thickness of the basal artery wall significantly increased $(\mathrm{P}<0.05)$, as compared with the blank and saline groups, in which no significant
\end{abstract}

Correspondence to: Dr Xian-Xi Tan, Department of Neurosurgery, First Affiliated Hospital of Wenzhou Medical College, 2 Fuxue Lane, Wenzhou, Zhejiang 325000, P.R. China

E-mail: tanxiangxi_txx@163.com

Key words: subarachnoid hemorrhage, delayed cerebral vasospasm, caveoion-1 alterations in the wall thickness and the inner perimeter of the basal artery pipe were detected. As detected by immunofluorescence and western blot analysis, the expression levels of caveolin-1 protein significantly decreased in the day 7 of SAH group, as compared with the blank and saline groups $(\mathrm{P}<0.01)$, in which no significant alterations were detected. Caveolin-1 mRNA expression levels significantly increased at the day 7 in the SAH group, as compared with the blank and the saline groups $(\mathrm{P}<0.01)$, as detected by RT-qPCR. Furthermore, significant differences were detected at day 14 in the $\mathrm{SAH}$ group, as compared with the blank and the saline groups $(\mathrm{P}>0.05)$, in which no significant alterations were detected. Therefore, the results of the present study demonstrated that caveolin-1 protein was downregulated in the basilar artery of a rat modeling SAH, which may be associated with DCVS. This suggested that caveolin-1 may be a potential target for the treatment of DCVS.

\section{Introduction}

Subarachnoid hemorrhage $(\mathrm{SAH})$ is a common cerebrovascular accident which is associated with high morbidity. SAH can be divided into spontaneous and traumatic SAH (1). The annual incidence of spontaneous SAH is $>10.5 / 100,000$ individuals per year and, since the majority of spontaneous SAH cases are induced by aneurysm rupture, the annual incidence of aneurysm rupture-induced spontaneous SAH is $6-35.5 / 100,000$ individuals per year (2). In recent years it has been demonstrated that the incidence of SAH is increasing (3). The American Heart Association demonstrated that the total mortality of SAH has reached $25 \%(4,5)$. Spontaneous SAH often results in numerous complications, of which cerebral vasospasm (CVS) is the most severe (6). CVS occurs due to the continuous contraction of one or several areas of smooth muscle in the intracranial vasculature, or vascular injury induced by sanguis stimulation, which leads to changes in the luminal morphological and intracranial vascular stenosis or spasm, whose incidence is $30-90 \%$ (7). According to clinical presentation, CVS may be divided into two types, early brain injury (EBI) and delayed cerebral vasospasm (DCVS) (8). EBI predominantly occurs in patients several minutes to half an hour post-SAH, as the blood which directly and mechanically stimulates the intracranial vasculature flows into cerebrospinal fluid (CSF) following SAH (8). 
DCVS predominantly occurs at 4-15 days post-SAH. DCVS often reaches vasospasm fastigium after 7-10 days, ultimately leading to severe ischemia of the local brain tissue, $\mathrm{d}$ (elayed ischemic injury or cerebral infarction, which are the predominant causes of morbidity and mortality in patients with DCVS (9). Patients with DCVS require long-term care and active nursing, therefore they are an increased burden on their family and limited medical resources, and suffer from decreased quality of life. Eventually, this may impede the development of social economic construction (10).

The occurrence of DCVS is associated with multiple factors and procedures. Previous studies have demonstrated that numerous factors influence the occurrence mechanism of DCVS, such as hemolysate, inflammatory response and endothelial dysfunction, which are associated with the occurrence of DCVS following SAH (11-13). The stabilization of endothelial cells has a key role in the normal physiological function of the human body. Vascular endothelial cells are cable of generating various vasoactive substances, including nitrogen oxide and prostacylin, which can dilate blood vessels, and endothelin (ET), arachidonic acid and cyclooxygenase, which can constrict vasculature (14). A previous study has investigated the dysfunction of vascular endothelin cells (15).

Caveolin is a type of integral membrane protein with a molecular weight of $17-24 \mathrm{kD}$, and is located on the inner surface of the alveolar cell membranes (16). Three members of the caveolin family, caveolin-1, caveolin-2 and caveolin-3, have previously been cloned (17). Although the structure and function of the caveolin gene family is highly conserved in mammals, the cellular distribution is diverse. Caveolin-1 is highly expressed in adipocytes, endotheliocytes and fibrocytes $(18,19)$. Caveolin can form complexes by combining with various signal molecules in order to regulate the activated state of numerous key signaling molecules and control transmembrane signal transduction (20). Furthermore, caveolin participates in vital cell activities in numerous cells, including endocytosis, the transportation of cholesterol, cytomembrane assembly and signal transduction (21-24).

Caveolin-1, which has a molecular weight of $21-24 \mathrm{kD}$, is associated with cholesterol homeostasis, molecular transportation and transmembrane signal transduction. In particular, a previous study has demonstrated that caveolin-1 has a role in signal transduction, the pathological and biological processes (25) of cell differentiation, proliferation, tumorigenesis, myopathy, angiopathy, neurodegenerative disorders and senility. Caveolin is a repository of calcium ions and is key for its transportation. Furthermore, previous studies have demonstrated that endothelial nitric oxide synthase (eNOS), which is a key signaling molecule associated with the precise regulation and control of calcium ions, is assembled around caveolin (26). Jasmin et al (26) have previously utilized caveolin knockout mice models in order to investigate the function of caveolin in cerebral ischemic injury. In addition, Jasmin et al (26) demonstrated that caveolin-1 gene knockout resulted in an increase in the cerebral infarct volume, as compared with the wild type, and the speed of endotheliocyte proliferation of the wild type mice with cerebral ischemia markedly increased, as compared with the caveolin-1 gene knockout mice. Furthermore, the eNOS levels of wild type mice with cerebral ischemia increased, whereas no notable alterations were detected in the caveolin-1 gene knockout mice. Therefore, these results demonstrated that cerebral ischemia induced an increase in the number of endotheliocytes and the expression of genes associated with angiogenesis was impaired by cerebral ischemia in mice with caveolin-1 knockout; therefore, the number of apoptotic cells increases. Using a mouse model of cerebral ischemia induced by arterial occlusion, Shen et al (27) investigated the function of eNOS in the regulation of caveolin-1. The results demonstrated that NO regulates the expression of caveolin-1, and reduced caveolin-1 expression is associated with the generation of $\mathrm{NO}$ in the ischemic brain. These previous studies demonstrated that caveolin-1 may serve a crucial function in the pathogenesis of cerebral ischemia and participates in the regulation of physiological mechanisms following cerebral ischemia $(26,27)$. Furthermore, it has previously been demonstrated that caveolin-1 is associated with various types of vascular disease, including atherosclerosis and hypertension, indicating that caveolin-1 may be associated with the differentiation of vascular endothelial cells (15). As endothelial cells have abundant cell membrane alveoli and caveolin-1, the authors of the present study hypothesize that caveolin-1 may be correlated with DCVS following SAH. To the best of our knowledge, the present study is the first to investigate whether caveolin-1 is associated with the development of SAH-induced DCVS.

Using a mouse model of SAH, generated by suboccipital pool double hemorrhage injection, the present study investigated the pathogenesis of DCVS by observing alterations in the levels of caveolin-1 in the basilar arteries of mice with DCVS following SAH. The results of the present study may provide novel theories for the future treatment of patients with DCVS following SAH.

\section{Materials and methods}

Experimental animals. A total of 150 clean-grade male Sprague-Dawley mice, weighing $300 \pm 10 \mathrm{~g}$ and aged 8-10 weeks, were purchased from Shanghai Slyke Experimental Animals, Co., Ltd. (SCXK 2007-0005; Shanghai, China). Ethical approval was obtained from the Ethical Committee of the First Affiliated Hospital of Wenzhou Medical College (Wenzhou, China).

Apparatus. P627 microscale pipettor; Thermostat (4304057; both Shanghai Medical Instrument Factory, Shanghai, China); Electronic analytical balance (ESJ182-4; Hangzhou Medical Equipment Factory, Hangzhou, China); Optical microscope (P7220; Thermo Fisher Scientiifc, Inc., Waltham, MA, USA), Fluorescence microscope (AF6000; Leica Microsystems GmbH, Wetzlar, Germany); Operating microscope (HR900A/2-11; Germany); Mice stereotaxic apparatus (YH51500D; Wuhan, China); Dural refrigerator (BCD-575WDBI; Haier Group, Qingdao, China); Micrograph system (Leica Microsystems GmbH); Thermostat water bath (W1933; Shanghai Cairrao Apparatus Company, Shanghai, China); T-Gradient polymerase chain reaction (PCR) amplifier (Biometra GmbH, Göttingen, Germany); Sigma 1-14 centrifuge (Sigma Laborzentrifugen $\mathrm{GmbH}$, Osterode am Harz, Germany); and concentrator table (TP22; Wuhan, China). 
Reagents. Chloral hydrate (302-17-0; Wuhan Boster Biological Technology, Ltd., Wuhan, China); paraformaldehyde (Z-P193265; Sinopharm Chemical Reagent Co., Ltd., Shanghai, China); rabbit polyclonal caveolin-1 primary antibodies (ab2910; Abcam, Cambridge, MA, USA); protein inhibitors (85-00-4975-03; Beijing Aolilaiyin Technology Co., Ltd., Beijing, China); peroxidase-AffiniPure goat anti-rabbit (111-035-003) and goat anti-mouse (115-035-003; both Jackson ImmunoResearch Laboratories, Inc., West Grove, PA, USA) immunoglobulin $(\mathrm{Ig}) \mathrm{G}(\mathrm{H}+\mathrm{L})$ fluorescent secondary antibodies; and goat anti-human IgG secondary antibodies (ZF-0308; Shanghai Boyun Chemical Reagent Co., Ltd., Shanghai, China). GAPDH (TA-08; Zhongshan Jinqiao, Beijing, China); enhanced chemiluminescence (ECL) kit (sc-2048; Cell Signaling Technology, Inc., Danvers, MA, USA). TRIzol kit (15596-018); caveolin-1 mRNA primer design; TaqMan DNA polymerase (4405495), diethylpyrocarbonate-treated water and 4',6-diamidino-2-phenylindole (62247) (all Invitrogen; Thermo Fisher Scientific, Inc.). DNase I kit (D8071-25; Solarbio Science \& Technology Co., Ltd., Beijing, China). Reverse transcription-quantitative PCR Master Mix (QPK-201; Toyobo Co., Ltd., Osaka, Japan). Finland reagent; hematoxylin and eosin (00-8011 and E-18); anti-fluorescence quencher (P0126); bicinchoninic acid assay (BCA) kit (P0011); ammonium persulfate (ST005); 30\% acrylamide; $10 \%$ sodium dodecyl sulfate (SDS); 1 M Tris- $\mathrm{HCl}$ (pH 6.8); $1.5 \mathrm{M}$ Tris-HCl (pH 8.8); tetramethylethylenediamine (ST066); phenylmethylsulfonyl fluoride (ST506); horseradish without IgG secondary antibodies marked (P0203); polyvinylidene difluoride (PVDF) membrane (A0793); and Tween-20 (ST825; all Biyuntian Nantong, Jiangsu, China).

Primary reagent compounds. i) 10\% Chloral hydrate: $10 \mathrm{~g}$ Chloral hydrate powder was supplemented with $100 \mathrm{ml}$ distilled water, incubated in a $55^{\circ} \mathrm{C}$ water bath unit and discontinuously stirred until the solution was clear. Solution was maintained in the dark. ii) 4\% Paraformaldehyde: $40 \mathrm{~g}$ Paraformaldehyde was supplemented with $100 \mathrm{ml}$ phosphate-buffered saline (PBS; $0.1 \mathrm{~mol} / \mathrm{l})$, discontinuously stirred until the solution was clear and the $\mathrm{pH}$ value was subsequently adjusted to 7.4 prior to preservation at $4^{\circ} \mathrm{C}$. iii) $5 \mathrm{X}$ Tris-buffered saline (TBS): $20.375 \mathrm{~g}$ Tris-HCl; $22.5 \mathrm{~g}$ sodium chloride, double-distilled water; and 1X TBS: $100 \mathrm{ml}$ TBS $(5 \mathrm{X})+400 \mathrm{ml}$ double-distilled water. iv) TBS with Tween-20 (TBST): $500 \mu 1$ Tween-20 + 500 ml TBS (1X). v) 10\% SDS-polyacrylamide gel electrophoresis (SDS-PAGE): $15.1 \mathrm{~g}$ Tris; $72 \mathrm{~g}$ glycine, $5 \mathrm{~g} \mathrm{SDS}, 500 \mathrm{ml}$ double-distilled water; $1 \%$ SDS-PAGE: $100 \mathrm{ml}(10 \mathrm{X})+900 \mathrm{ml}$ double-distilled water. vi) $10 \mathrm{X}$ Transfer buffer: $15 \mathrm{~g}$ Tris, $72 \mathrm{~g}$ glycine, $5 \mathrm{~g}$ SDS, $500 \mathrm{ml}$ double-distilled water; and $1 \mathrm{X}$ transfer buffer: $100 \mathrm{ml}(10 \mathrm{X})+200 \mathrm{ml}$ carbinol $+700 \mathrm{ml}$ double-distilled water. vii) $5 \%$ sealing milk: $5 \mathrm{~g}$ skim milk powder $+100 \mathrm{ml}$ TBST liquid. viii) ECL solution: ECL solution A + ECL solution B (mixed 1:1).

Experimental groups. Mice were allocated into three groups: Blank group $(n=14)$, the saline group $(n=56)$ and the operational group $(n=80)$. The normal saline and operational groups were further divided into four subgroups: Days 3, 5, 7 and 14 post-SAH. The mice in the operational group were injected autologous arterial blood into subarachnoid space. The saline group was injected equivalent saline into subarachnoid space. The blank group did not receive any treatment.

Murine model of SAH-induced DCVS. In the present study a model of SAH was created using the method of double hemorrhage of cisterna magna, as previously described (28). Mice were intraperitoneally anesthetized using $10 \%$ chloral hydrate $(3 \mathrm{ml} / 100 \mathrm{~g})$ and fixed in the prone position. Following disinfection of the preserved skin using $75 \%$ ethanol, the fascia muscle was incised along the middle wire, using an operational microscope. Mice were subsequently placed in the supine position and the skin of the inguinal region was disinfected prior to the incision of the skin to expose the femoral artery, which was subsequently separated and fixed. Following these procedures, $0.01 \mathrm{ml}$ heparin $(25 \mathrm{U})$ was injected into the artery using a hollow 1-ml needle and mice were fixed into the prone position with their heads bent forward to extract $0.10 \mathrm{ml} / 100 \mathrm{~g}$ autologus arterial blood by weight. Subsequently, autologus arterial blood was slowly injected into the cisternas magna (speed, $0.1 \mathrm{ml} / \mathrm{min}$ ) from the foramen occipital magnum using a needle inserted at $1 \mathrm{~mm}$. A 'breakthrough' sensation was detected when the needle was inserted into the atlantooccipital membrane and the puncture was immediately sealed with a gelatin sponge. After hemostasis, the muscular layer and skin were sutured layer-by-layer. Subsequently, mice were placed in a dorsal elevated position for $30 \mathrm{~min}$, and placed in a cage. After $48 \mathrm{~h}$, blood was harvested from the femoral artery and injected for the second time. The method remained the same. A total of $24 \mathrm{~h}$ after the second injection was regarded as day 1 post-SAH, and so on.

Grading of neurological impairment in the SAH group. Neurological impairment was assessed according to grading system proposed by Bederson (29). Grading was conducted on days 3,5 and 14 post-SAH, according to the condition of the tail, particularly its bend and whirl, and resistance of its bilateral forelegs, and the condition of the whirl. Scoring was classified as follows: 0, Normal; 1, mild neurological impairment; 2, moderate neurological impairment; and 3, severe neurological impairment.

\section{Specimen collection}

New specimen collection. Following establishment of the mouse model of DCVS, mice were anesthetized using $3 \mathrm{ml} / 100 \mathrm{~g}$ chloral hydrate (10\%) on days 3,5 and 14 post-SAH, according to the sub-groupings. Following this, the respective mouse brains were harvested, placed on an iced plate in order to separate basilar artery and rapidly preserved in liquid nitrogen.

Preparation of paraffin specimen. The abdominal skin of the mice was cut off, the ensisternum was lifted using a vessel clamp, and the ribs were cut along the two sides of sternum using scissors in order to expose the heart, ventriculus sinister, and right auricular. A 24-gauge transfusion needle was inserted into the ventriculus sinister and, simultaneously, using the hemostatic forceps to clamp the transfusion needles and the left ventricular wall, normal saline was quickly infused. Immediately, scissors were used to cut the right auricular in order to release the blood until the effluent was 
clear. Following this, $300 \mathrm{ml}$ paraformaldehyde (4\%), which had been prepared previously, was injected quickly at first then slowly until the heads and necks of the mice stiffened. Upon completion of the injection, the mice were immediately decapitated and the scalps were simultaneously removed. The skulls were subsequently removed and the brainstems, which contains basal arteries, were respectively harvested. All the mice brainstems were fixed in $4 \%$ paraformaldehyde and, following $24 \mathrm{~h}$, were paraffin embedded. The basal artery brainstem specimens were trimmed to $10 \times 5 \times 4 \mathrm{~mm}$ and placed in the embedding boxes. Specimens were processed according to the following protocol: Overnight incubation with $75 \%$ ethyl alcohol; once with $85 \%$ ethyl alcohol for $2 \mathrm{~h}$; twice for hour with $95 \%$ ethyl alcohol; twice for 45 min with absolute ethyl alcohol to dehydrate; twice for $45 \mathrm{~min}$ with xylene to make the cells transparent; wax I overnight; wax II for $2 \mathrm{~h}$; and the wax was subsequently injected into the specimen. After the wax had cooled down, the specimen was sliced using the microtome, with the slicing knife parallel with the transverse section to ensure with each section was $4 \mu \mathrm{m}$-thick. Each specimen was subsequently cut into 10 continuous pieces and subjected to HE staining and immunofluorescence analysis, respectively.

HE staining. Staining with HE was performed in order to measure the interfacial inner diameter of the basilar artery and the thickness of the vessel wall of the basilar artery. Five section pieces were selected from each specimen, were dried for $60 \mathrm{~min}$ at $65^{\circ} \mathrm{C}$ and stained as follows: Deparaffinization with xylene twice for 5 min; hydration using an alcohol gradient: $100 \%$ Ethyl alcohol twice for $5 \mathrm{~min}, 95 \%$ ethyl alcohol once for $5 \mathrm{~min}, 90 \%$ ethyl alcohol once for $5 \mathrm{~min}, 70 \%$ ethyl alcohol once for $5 \mathrm{~min}$, and washing three times with distilled water for $5 \mathrm{~min}$; staining with hematoxylin for $10 \mathrm{~min}$, incubation at $37^{\circ} \mathrm{C}$ for $3 \mathrm{~min}$ in warm water to ensure the sections are stained blue; washing with running water for $10 \mathrm{~min}$; followed by counterstaining with eosin for $5 \mathrm{~min}$; $95 \%$ ethyl alcohol twice for $5 \mathrm{~min}$, xylene twice for $10 \mathrm{~min}$, and subsequent mounting using neutral gum. The interfacial inner diameter of basilar artery was calculated using the Image Pro Plus 5.1 image analyzing system (Media Cybernetics, Inc., Rockville, MD, USA) and the thickness of the vessel wall was measured and the mean values were calculated. Following dewaxing, the sections were treated with $0.3 \% \mathrm{H}_{2} \mathrm{O}_{2}$ in methanol for $10 \mathrm{~min}$, washed with distilled water supplemented with $0.01 \mathrm{M}$ citrate solution ( $\mathrm{pH}$ 6.0) and heated in an oven for $10 \mathrm{~min}$. Following cooling to $25^{\circ} \mathrm{C}$, the sections were washed three times with PBS prior to immunohistochemical analysis.

Immunofluorescence analysis. Five sections were taken from each specimen and the section was dried for $60 \mathrm{~min}$ at $65^{\circ} \mathrm{C}$; deparaffinized with xylene twice for $5 \mathrm{~min}$; hydrated using an alcohol gradient (100\% ethyl alcohol twice for $5 \mathrm{~min}, 95 \%$ ethyl alcohol once for $5 \mathrm{~min}, 90 \%$ ethyl alcohol once for $5 \mathrm{~min}$, $70 \%$ ethyl alcohol once for $5 \mathrm{~min}$, and washed three times with PBS water for $5 \mathrm{~min}$ ). Antigen retrieval was performed using sodium citrate, the sections were washed three times with PBS for $5 \mathrm{~min}$, immersed in $3 \% \mathrm{H}_{2} \mathrm{O}_{2}$ to wipe off the peroxidase, sealed using serum, incubated overnight with rabbit polyclonal caveolin-1 primary antibodies antibody $(1: 1,000)$. Following this, the sections were washed three times with PBS water for
$5 \mathrm{~min}$ and incubated for $1.5 \mathrm{~h}$ with secondary fluorescence antibody $(1: 1,000)$ in the dark room. Following washing three times with PBS water for 5 min, 4',6-diamidino-2-phenylindole, and fluorescence agent quenching mounting, the sections were observed under a fluorescence microscope.

\section{Western blot analysis}

Extraction and measurement of protein. Protein extraction was performed as follows, $50 \mathrm{mg}$ cryopreserved basilar artery was placed in the grinding rod and supplemented with $500 \mu 1$ organization lysate and $5 \mu 1$ proteinase inhibitor prior to full grinding on ice for $30 \mathrm{~min}$ The liquid was collected, placed in 1.5-ml non-enzyme tubes, centrifuged for $5 \mathrm{~min}$ at $12,500 \mathrm{x}$ g, prior to supplementing the dissolution liquid with $150 \mu 1$ protein preserving liquid, which can be adjusted by the precipitation capacity. After repeatedly blowing and beating the sediment solution, the dissolution was placed in a $0.5-\mathrm{ml}$ centrifuge tube and $20-25 \mu \mathrm{l}$ was preserved at $80^{\circ} \mathrm{C}$. Protein concentration was measured using a BCA kit, according to the manufacturer's protocol, where the solubility of the protein was measured using a microplate reader.

Western blot analysis. Proteins were separated by $10 \%$ SDS-PAGE, which was selected according to the molecular weight of the protein to be measured, caveolin-1. Following albuminous degeneration $\left(100^{\circ} \mathrm{C}\right.$ for $\left.5 \mathrm{~min}\right), 20 \mu \mathrm{l}$ protein was loaded into the respective wells using a microscale pipette, and an equal quantity of buffer solution was subsequently loaded in order to avoid edge effects. Electrophoresis was run in $1 \mathrm{X}$ liquid at $35 \mathrm{~mA}$ for $90 \mathrm{~min}$, until the leading edge of the dye had migrated to the bottom of the gel. Proteins were transferred to a PVDF membrane and were rinsed using double-distilled water for $5 \mathrm{~min}$. The membrane was transferred at $350 \mathrm{~mA}$ for $60 \mathrm{~min}$ in the $1 \mathrm{X}$ transferring membrane liquid. In order to seal the PVDF membrane, it was removed, washed with deionized water and incubated with $5 \%$ skim milk for $2 \mathrm{~h}$ on the concentrator table. Subsequently, the membrane was washed three times with TBST for $10 \mathrm{~min}$ and incubated with caveolin-1 and GAPDH primary antibodies $(1: 1,000)$ overnight at $4^{\circ} \mathrm{C}$. Following this, the membrane was again washed three times with TBST for $10 \mathrm{~min}$ and incubated with goat anti-rabbit secondary antibodies $(1: 2,500)$, for $2 \mathrm{~h}$ at $25^{\circ} \mathrm{C}$ on the concentrator table. The membrane was washed three times with TBST for $10 \mathrm{~min}$ prior to development and exposure. According to the ECL protocol, ECL solutions A and $\mathrm{B}$ were mixed (ratio, 1:1), and incubated with the PVDF membrane in the dark room to develop for 3-5 min prior to exposure. Data were analyzed using ImageJ protein grayscale software (National Institutes of Health, Bethesda, MA, USA).

\section{$P C R$}

Extraction, concentration determination and reverse transcription of RNA. RNA was extracted by grinding 50-100 mg tissue into powder in liquid nitrogen and subsequently injecting $1 \mathrm{ml} \mathrm{TRIzol}$ prior to further grinding for $15 \mathrm{~min}$. Following this, the homogenate was transferred into an Eppendorf (EP) tube and left to dissociate for $5 \mathrm{~min}$ at room temperature prior to centrifugation $12,000 \times \mathrm{g}$ for $10 \mathrm{~min}$ at $4^{\circ} \mathrm{C}$. Using a new EP tube, the supernatant was collected and supplemented with chloroform at the ratio of 0.2:1 $\mathrm{ml}$ TRIzol. The EP tube was 
A

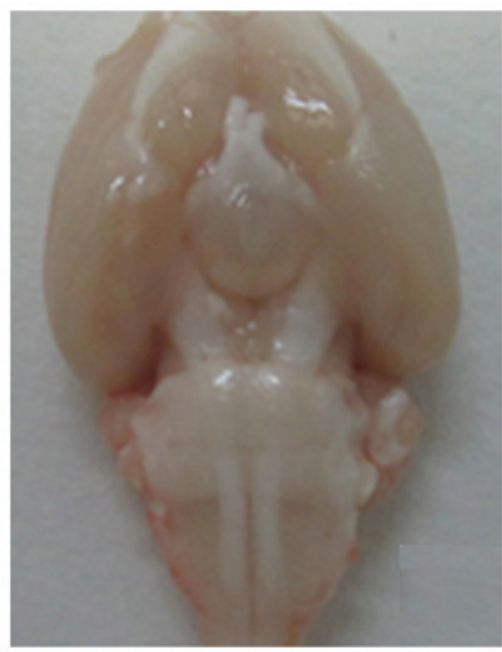

B

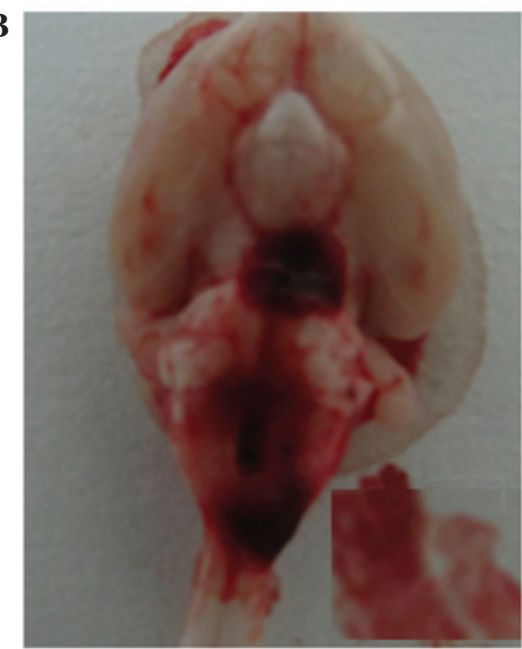

Figure 1. Fresh brain tissue harvested from the (A) blank and (B) experimental groups.

shaken for $15 \mathrm{sec}$ by hand prior to incubation for $3 \mathrm{~min}$ at room temperature and subsequent centrifugation at $12,000 \mathrm{x} \mathrm{g}$ for $15 \mathrm{~min}$ at $4^{\circ} \mathrm{C}$. The supernatant was transferred to a new EP tube, supplemented with isopropanol at the ratio of $0.5 \mathrm{ml}$ to $1 \mathrm{ml} \mathrm{TRIzol}$, incubated for $10 \mathrm{~min}$ at room temperature and subsequently centrifuged at $12,000 \mathrm{x}$ g for $10 \mathrm{~min}$ at $4^{\circ} \mathrm{C}$. After discarding the supernatant, the pellet was washed with $75 \%$ ethyl alcohol at the ratio of $\leq 1 \mathrm{ml}$ ethyl alcohol to $1 \mathrm{ml}$ TRIzol. Following shaking, the supernatant was discarded and the sedimentary RNA was left to dry naturally at room temperature. Once dry, the pellet was suspended in 20-40 $\mu 1$ diethylpyrocarbonate-treated non-enzyme water and stored at $-70^{\circ} \mathrm{C}$.

RNA concentration was determined by suspending $2 \mu \mathrm{l}$ RNA in $98 \mu \mathrm{l}$ non-RNase water and centrifuging at $12,000 \times \mathrm{g}$ for $10 \mathrm{~min}$ at $4^{\circ} \mathrm{C}$, prior to measuring the optical density (OD) 260/280 value using a spectrophotometer ( $1 \mathrm{OD}=40 \mu \mathrm{g}$ RNA). When the OD 260/280 value of the RNA sample was detected as 1.8-2.0, the purity was regarded as high. In general, the normal concentration value was determined to be $1,000 \mathrm{ng} / \mathrm{ml}$, although when the concentration was deemed too high, it was attenuated by diluting the sample with an appropriate volume of non-RNase water and the measurement was repeated. Reverse transcription was performed using TaqMan DNA polymerase by initially placing RNA at $65^{\circ} \mathrm{C}$ for $51 \mathrm{~min}$, and immediately transferring the sample to ice to cool. Subsequently, the reverse transcription reaction was performed for $15 \mathrm{~min}$, followed by enzyme inactivation for 5 min. Upon completion, the subsequent cDNA underwent DNase treatment and was stored at $-20^{\circ} \mathrm{C}$.

RT-qPCR analysis. PCR reaction mixtures contained: $5 \mu \mathrm{l} \mathrm{SYBR}$ Green Realtime PCR Master Mix-Plus; $1 \mu 1$ Plus Solution; $1 \mu \mathrm{l}$ upstream and downstream primers $(10 \mu \mathrm{mol} / \mathrm{l})$, respectively; and $1 \mu \mathrm{l}$ template supplemented with sterile deionized water to a final reaction volume of $10 \mu \mathrm{l}$. Reaction conditions were as follows: $94^{\circ} \mathrm{C}$ for $3 \mathrm{~min}$, then $94^{\circ} \mathrm{C}$ for $5 \mathrm{sec}$, $60^{\circ} \mathrm{C}$ for $20 \mathrm{sec}$, all for 40 cycles. Mouse actin was used as the reference gene, against which the expression levels of the target genes were normalized to calculate the standard curves for the real-time quantification of the caveolin-1 target genes. The following primers were used: Caveolin-1, upstream 5'-GAC CCCAAGCATCTCAACGA-3' and downstream 5'-GCC ATAGGGATGCCGAA-GA-3'; and actin internal reference, upstream 5'-AGAGGGAAATCGTGCGTGAC-3' and downstream 5'-AGAGGTCTTTACGGATGT-CAACG-3'. In order to increase the credibility of the results, each specimen was repeated in 6 tubes, 3 of which were target genes, and the remainder were internal reference. RT-qPCR analysis of the target gene and internal reference was completed in triplicate in order to determine the mean $\mathrm{Cq}$ value. Relative mRNA expression levels of caveolin-1 were calculated by dividing the mean $\mathrm{Cq}$ of actin by the mean $\mathrm{Cq}$ of caveolin-1, according to the $2^{-\Delta \Delta \mathrm{Cq}}$ method.

Statistical analysis. Experimental data were statistically analyzed using SPSS 18.0 software (SPSS, Inc., Chicago, IL, USA). Data were expressed as the mean \pm standard deviation. Inter-group and intragroup comparisons were performed using Student's t-test. $\mathrm{P}<0.05$ was considered to indicate a statistically significant difference.

\section{Results}

Establishment of the murine model. During the observation period, 10 mice succumbed to SAH-induced DCVS in the day 3 $(n=3), 5(n=5)$ and $7(n=2)$ groups. No mortality was observed in the blank control and normal saline groups. In the experimental group, blood clots were observed around the basilar artery of the respective brainstems during the craniotomy (Fig. 1A and B). Furthermore, all mice in the SAH group had Bederson neurological severity scores $\geq 1$, which indicated the success of the model (Table I). No neurological impairment was detected in the blank and normal saline groups.

As detected by light microscopy, HE staining of the basilar artery demonstrated that the inner membranes of the basilar arteries appeared smooth in the blank and normal saline groups and the inner elasticity and integrity were maintained. In the SAH groups, the inner perimeter of the basilar artery was reduced, the thickness of the arterial wall of the basilar 
Table I. Bederson grading of neurological impairment in the mouse model of subarachnoid hemorrhage $(\mathrm{SAH})$ group $(\mathrm{n}=20)$.

\begin{tabular}{|c|c|c|c|c|c|}
\hline \multirow[b]{2}{*}{ Duration of SAH } & \multicolumn{4}{|c|}{ Bederson grade of neurological impairment } & \multirow[b]{2}{*}{ Mortality $(\%)$} \\
\hline & 0 & 1 & 2 & 3 & \\
\hline 3 days & 0 & 9 & 8 & 0 & 15 \\
\hline 5 days & 0 & 3 & 6 & 6 & 25 \\
\hline 7 days & 0 & 3 & 11 & 4 & 10 \\
\hline 14 days & 0 & 14 & 6 & 0 & 0 \\
\hline
\end{tabular}

No neurological impairment was detected in the blank and normal saline groups.

Table II. Internal diameter $(\mu \mathrm{m})$ of the basilar artery in the blank, normal saline and subarachnoid hemorrhage (SAH) groups.

\begin{tabular}{lccr}
\hline Duration of SAH & Blank group & Normal saline group & SAH group \\
\hline 3 days & $1,002 \pm 0.24$ & $1,001 \pm 0.45$ & $701 \pm 8.34$ \\
5 days & $1,003 \pm 0.56$ & $1,003 \pm 0.32$ & $686 \pm 6.23$ \\
7 days & $1,003 \pm 0.29$ & $1,002 \pm 0.26$ & $638 \pm 5.68$ \\
14 days & $1,001 \pm 0.31$ & $1,002 \pm 0.67$ & $698 \pm 4.65$ \\
\hline
\end{tabular}
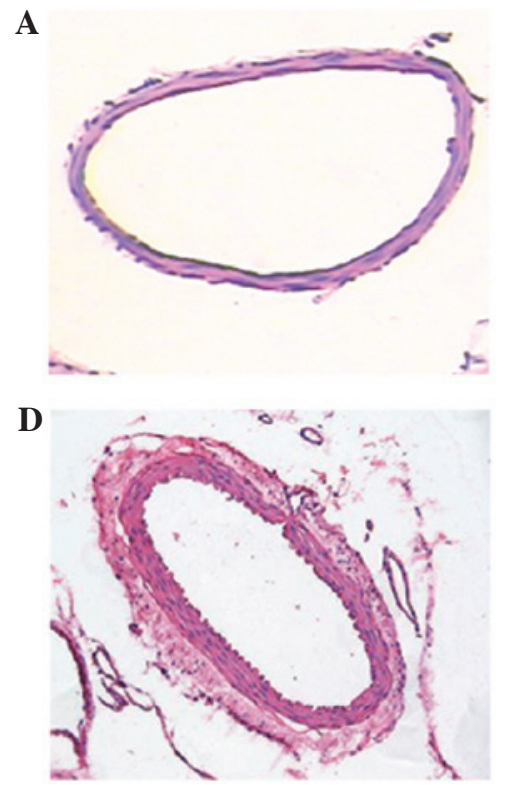

B

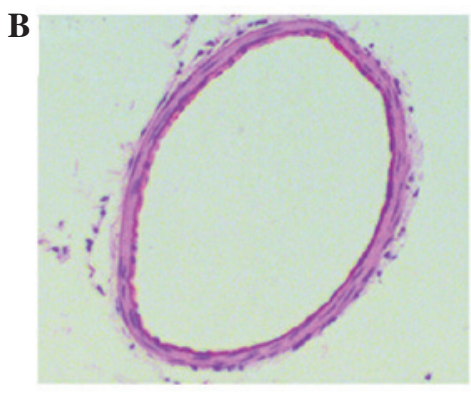

E

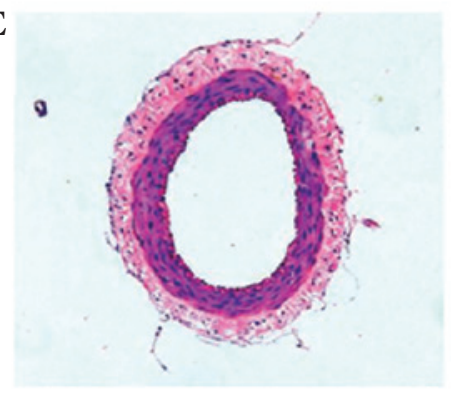

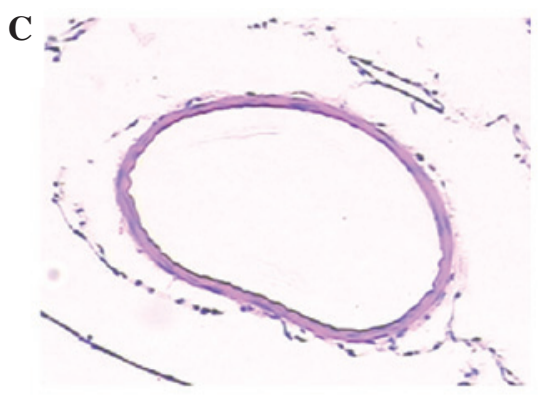

$\mathbf{F}$

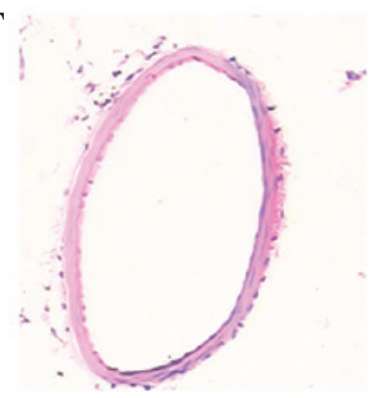

Figure 2. Hematoxylin and eosin staining of the basilar artery of a murine model of subarachnoid hemorrhage (SAH)-induced delayed cerebral vasospasm, as detected by a light microscope (magnification, 20x). (A) Blank group, (B) normal saline group, (C) experimental group subjected to SAH for 3, (D) 5, (E) 7 and (F) 14 days. The inner membranes of the basilar arteries in the blank and normal saline groups appeared smooth and the inner elastic force maintained integrity. In the SAH groups, the inner perimeter of the basilar artery was reduced, the thickness of the arterial wall of the basilar artery increased seen from the light microscope and its inner membrane appeared to be buckling, as compared with the blank and normal saline groups.

artery increased under the light microscope and its inner membrane appeared to be buckling, as compared with the blank and normal saline groups (Fig. 2).

SAH significantly reduces the internal diameter of the basilar artery. The internal diameter of the basilar artery was measured in all the groups at various time points (Table II). Across all durations of SAH, the internal diameter of the basilar artery was significantly reduced in the SAH group at the same time point, as compared with the normal saline and blank groups $(\mathrm{P}<0.01)$. The t-values for the $\mathrm{SAH}$ group, and the normal saline and blank groups were 9.72 and 10.13, respectively (data not shown). No significant differences were detected between the normal saline and blank groups (t-value, 3.49; $\mathrm{P}>0.05$ ).

SAH significantly increases the wall thickness of the basilar artery. The wall thickness of the basilar artery was measured 
Table III. Wall thickness $(\mu \mathrm{m})$ of basilar artery in the blank, normal saline and subarachnoid hemorrhage (SAH) groups.

\begin{tabular}{lccc}
\hline Duration of SAH & Blank group & Normal saline group & SAH group \\
\hline 3 days & $9.80 \pm 0.34$ & $9.26 \pm 0.13$ & $22.68 \pm 0.36$ \\
5 days & $9.47 \pm 0.29$ & $9.43 \pm 0.20$ & $36.74 \pm 0.52$ \\
7 days & $9.58 \pm 0.27$ & $9.39 \pm 0.32$ & $64.86 \pm 0.31$ \\
14 days & $9.24 \pm 0.12$ & $9.35 \pm 0.19$ & $32.16 \pm 0.45$ \\
\hline
\end{tabular}

A
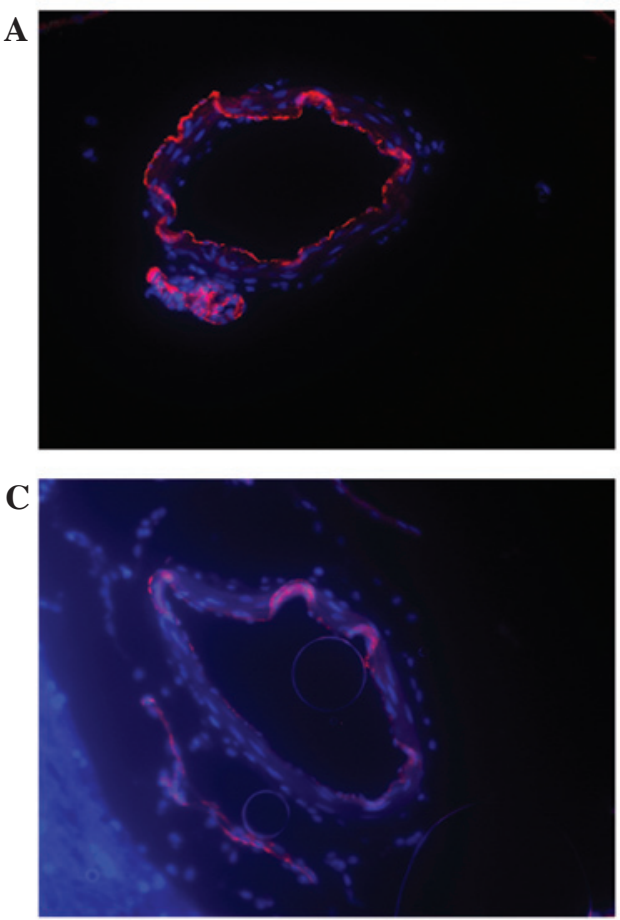

B

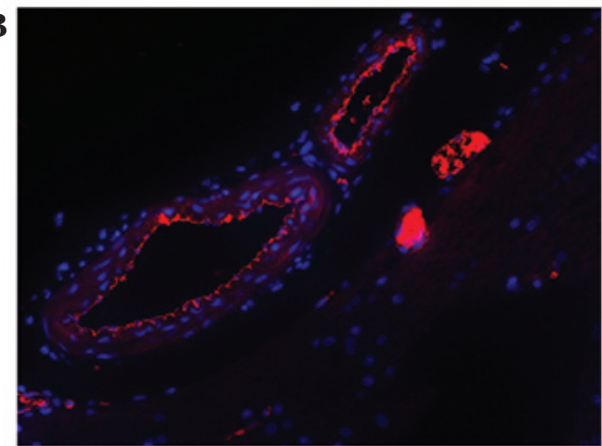

D

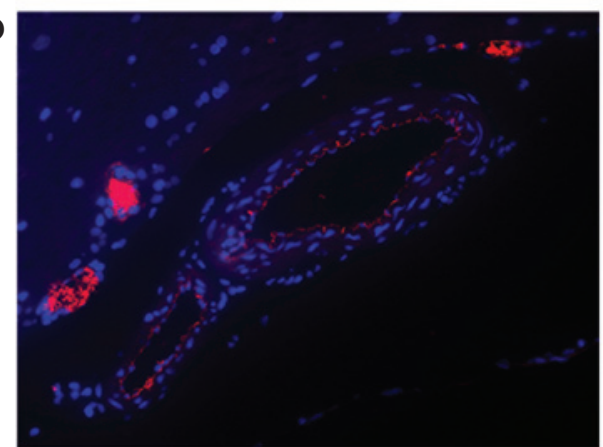

Figure 3. Immunofluorescence analysis of caveolin-1 expression in the basilar artery of a murine model of subarachnoid hemorrhage (SAH)-induced delayed cerebral vasospasm (DCVS) (magnification, 40x). (A) Blank group, (B) normal saline group and experimental groups subjected to SAH-induced DCVS for (C) 5 and (D) 7 days. Red fluorescence denotes caveolin-1 expression; whereas the blue denotes nuclear staining. Caveolin-1 expression in the endothelial cells of the basilar artery was significantly reduced $(\mathrm{P}<0.05)$ in the experimental group subjected to SAH-induced DCVS for 5 days, as compared with the blank and normal saline groups. Although caveolin-1 expression notably improved by the seventh day, the reduction remained significant on the seventh day, as compared with the blank and normal saline groups $(\mathrm{P}<0.05)$. No significant differences were detected between the normal saline and blank groups.

in all the groups at various time points (Table III). Across all durations of SAH, the wall thickness of the basilar artery was significantly increased in the SAH group at the same time point, as compared with the normal saline and blank groups. The t-values for the SAH group, and the normal saline and blank groups were 8.63 and 8.19 , respectively $(\mathrm{P}<0.01$; data not shown). No significant differences were detected between the normal saline and blank groups (t-value, 2.18; $\mathrm{P}>0.05$ ).

SAH significantly reduces the expression of caveolin-1 in the basilar artery, as detected by fluorescence immunoassay. Immunofluorescence analysis of caveolin-1 expression in the basilar artery was performed on all groups (Fig. 3). The results demonstrated that caveolin-1 expression levels in the endothelial cells of the basilar artery were markedly reduced in the experimental group subjected to SAH-induced DCVS for 5 days, as compared with the blank and normal saline groups. Although caveolin-1 expression notably improved by day 7 of $\mathrm{SAH}$, expression levels remained reduced, as compared with

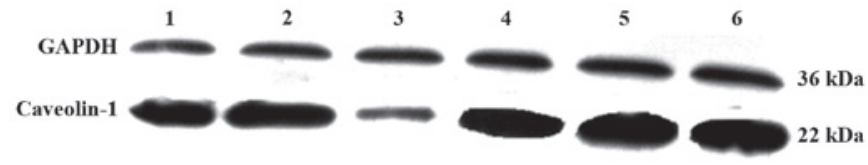

Figure 4. Western blot analysis of the expression of caveolin-1 in the basilar artery of a murine model of subarachnoid hemorrhage (SAH)-induced delayed cerebral vasospasm. GAPDH was used as an internal reference. 1, Day 3 of SAH group; 2, day 5 of SAH group; 3, day 7 of SAH group; 4, day 14 of SAH group, 5 , day 7 of the blank group; 6 , day 7 of the normal saline group. GAPDH, glyeraldehyde 3-phosphate dehydrogenase.

the blank and normal saline groups. No significant differences were detected between the normal saline and blank groups.

Western blot analysis for the detection of caveolin-1 protein expression in the basilar artery. The t-values of SAH group, and the normal saline and blank groups were 21.72 and 53.66, respectively, at each time point $(\mathrm{P}<0.01$; data not 


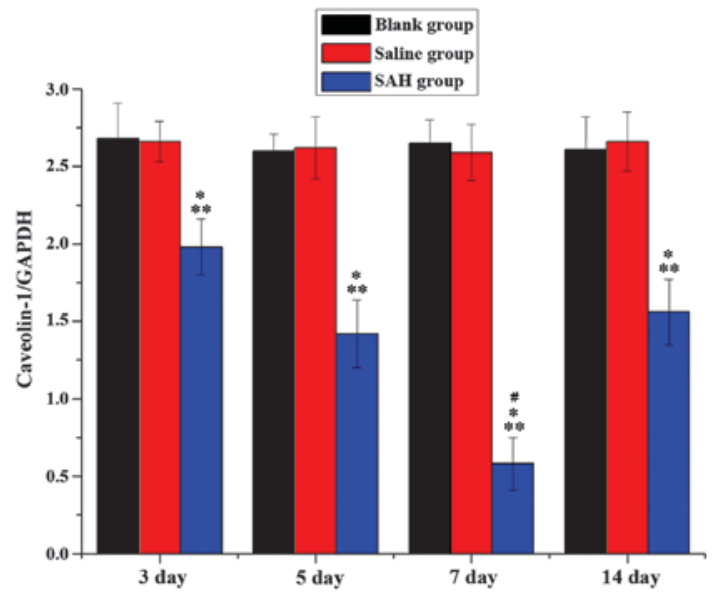

Figure 5. Relative expression levels of caveolin-1 protein, as demonstrated by grayscale analysis. SAH, subarachnoid hemorrhage; GAPDH, glyceraldehyde 3-phosphate dehydrogenase. " $\mathrm{P}<0.05$, vs. the blank group; ${ }^{* *} \mathrm{P}<0.05$, vs. the saline group; ${ }^{\prime} \mathrm{P}<0.05$, vs. the other time points.

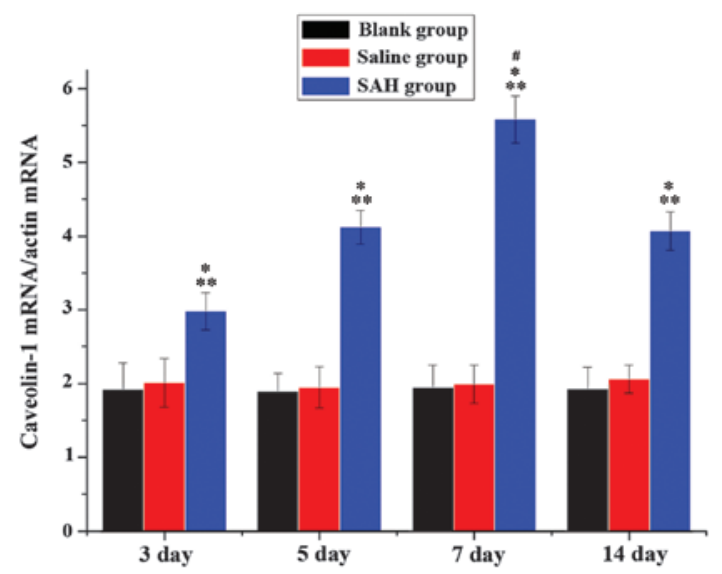

Figure 6. Reverse transcription-quantitative polymerase chain reaction analysis of the mRNA expression levels of caveolin-1 mRNA. Caveolin-1 mRNA expression levels were significantly upregulated across all time points in the SAH group, peaking on day 7 of SAH $(\mathrm{P}<0.05)$. No significant differences were detected between the blank and normal saline groups. SAH, subarachnoid hemorrhage. ${ }^{*} \mathrm{P}<0.05$, vs. the blank group; ${ }^{* *} \mathrm{P}<0.05$, vs. the saline group; ${ }^{\text {"N}} \mathrm{P}<0.05$, vs. the other time points.

shown). No significant differences were detected between the normal saline and blank groups (t-value, 2.18; $\mathrm{P}>0.05$ ). Caveolin-1 expression levels were significantly reduced in the SAH groups at all time points, as compared with the normal saline and blank groups (Figs. 4 and 5). When the SAH groups were compared, the reduction in caveolin-1 expression levels detected on the day 7 was significantly lower than the other SAH group time points.

RT-qPCR analysis of caveolin-1 mRNA expression. RT-qPCR analysis was performed to compare the relative mRNA expression levels of caveolin-1 in the normal saline, blank and SAH groups at various time points (Fig. 6). The results demonstrated that caveolin-1 mRNA expression levels were significantly increased across all time points in the SAH group, peaking on day 7 of SAH $(\mathrm{P}<0.05)$. No significant differences were detected between the blank and normal saline groups.

\section{Discussion}

SAH is a common cerebrovascular emergency. A total of 30\% patients with arterial aneurysm SAH will experience DCVS, which is a common complication of SAH that remains the predominant cause of patient morbidity and mortality (28). Cerebral angiospasm typically occurs on days 3-5 following SAH, peaks on days 7-10 and ceases after 2-3 weeks. Cerebral angiospasm causes delayed ischemic brain damage (30). Although a previous study has investigated the pathogenesis of DCVS in patients following SAH, the mechanisms underlying the pathogenesis of DCVS are yet to be elucidated (31).

DCVS is the most severe complication of SAH and is the predominant factor affecting the prognosis of patients post-SAH (32). Therefore, it is crucial that the underlying mechanisms of post-SAH DCVS are investigated and potential clinical treatment options are elucidated. However, a simple practicable animal model of post-SAH DCVS is required in order to further study the pathogenesis and clinical treatment of DCVS. The establishment of a good DCVS animal model is the key finding of the present study. Previous experimental animal models have been created to study DCVS via methods such as, puncturing the murine middle cerebral artery to induce SAH (33), mice pulvinar next blood injection and the recognized method of murine cisterna magna double hemorrhage injection which was used to establish the present mouse model of SAH (34). As a good DCVS animal model involves the production of a blood clot circling around basicranial blood vessel $(34,35)$, mice occipital bone double hemorrhage injection was adopted in the present study to form the most effective animal model possible. According to the grading system of neurological impairment proposed by Bederson (29), mice in the SAH day 3,5 and 7 experimental groups exhibited Bederson neurological severity scores $\geq 1$; whereas no neurological impairment was detected in normal saline and normal control groups. The SAH groups demonstrated varying degrees of neurological impairment, particularly in the day 5 and 7 SAH groups, where neurological impairment was graded as the most severe. This result is consistent with the time of the appearance of DCVS symptoms following SAH. Therefore, the results of the present study suggested that a mouse model of DCVS following SAH was successfully established, thus supporting the accuracy and objectivity of the experimental data.

DCVS following SAH is a complex pathological process, which is associated with vascular endothelial function impairment, shrinkage and proliferation of vascular smooth muscle, immune inflammatory reaction and gene regulation (36). It has previously been demonstrated that cerebrovascular endothelial cell damage is the key characteristic of cerebral artery pathology in patients following SAH (37). Thus, it may be associated with the pathogenic mechanism of DCVS. Endothelial cells dilate vessels by generating NO, whereas they constrict vessels via ET. Under normal pathology, there is a dynamic balance between the two to maintain homeostasis via the dilation and constriction of vessels (38). Disorder of this balance following SAH is a key factor in the pathogenesis of DCVS. In recent years, studies in this field have focused on NO, which is generated by eNOS and L-arginine, and is a key factor for the development of DCVS $(39,40)$. At the 
same time, the organism also generates a kind of inductive NOS. The generation of NO in vivo is controlled by a negative-feedback mechanism that is dependent on NO content, the feedback affection of NOS and methylate of L-arginine. $\mathrm{NO}$ adjusts vasodilatation via the cyclic guanosine monophosphate (cGMP) signaling pathway (41). When the endothelial cells secrete NO, NO acts on smooth muscle cells, activating guanylate cyclase in the inner cytoplasm to generate cGMP, which facilitates the opening calcium channels on the endoplasmic reticulum membrane (42). Calcium ions subsequently flow into the endoplasmic reticulum, inducing the dilation of smooth muscle. As a result, oxygen and hemoglobin consumption increases and the generation of endogenous NO decreases, which in turn decreases the bioavailability of NO on endothelial cells, leading to vasoconstriction and stenosis of the lumen (43). ET-1 is released from the vestibular nucleus in endothelial cells and is activated by receptor binding to generate diacyl glycerol (DAG), leading to vasoconstriction via DAG-protein kinase C (PKC) (44). Caveolin-1 is localized between endothelial cells and vascular smooth muscle cells and is a key constitutive protein for the formation of caveolae. The oligomerization domain and caveolin scaffolding domain (CSD) are key to the structure and function of this area. On endothelial and vascular smooth muscle cells, caveolin-1 prevents PKC from activating CSD its by preventing interaction and subsequent enzymatic activity (45). Therefore, this can results in a reduction in the concentration of the ET compound and subsequent vasodilatation. Furthermore, caveolin-1 is capable of attenuating the excitation of downstream protein molecules to reduce the release of eNOS, which weakens the negative feedback NO release by combining CSD microcell and eNOS (46). This increases the release of NO, leading to vasodilatation (2). Shen et al (28) demonstrated that the loss of caveolin-1 is associated with the generation of NO in the brain.

The present study demonstrated that the inner tube of the basilar artery significantly narrowed on day 3 following $\mathrm{SAH}$, peaking on day 7 , and subsequently attenuating on day 14 , as compared with day 7 . All the results were obtained via observation of inner diameter and thickness of the basilar artery. The results of the present study also indicated that the smooth muscle layer became thicker on day 5 post-SAH, once again peaking on day 7 and attenuating by day 14 . Caveolin-1 expression in the basilar artery was detected using immunofluorescence and western blot analyses; the results of which demonstrated that caveolin-1 protein expression significantly reduced by day 5 following $\mathrm{SAH}(\mathrm{P}<0.05)$, as compared with the normal saline and blank groups. Caveolin-1 mRNA expression levels were detected using RT-qPCR. The results demonstrated that the mRNA expression level of caveolin-1 significantly increased following $\mathrm{SAH}$, and peaked on day 7, as compared with the blank and normal saline groups $(\mathrm{P}<0.01)$. These results were consistent with previous findings (47) and demonstrated similar time-related development of symptoms in patients with SAH-induced DCVS. Therefore, the results of the present study suggested that caveolin-1 may be crucially involved in the development of DCVS following SAH.

In conclusion, the method of cisterna magna double hemorrhage injection may be used to successfully establish a model of DCVS following SAH. Furthermore, the downregulation of caveolin-1 expression detected in the basilar artery of mice with DCVS following SAH suggested that caveolin-1 may be associated with the development of DCVS following SAH. Therefore, intervening and regulating caveolin-1 and its associated mechanisms may be a potential target for the treatment of DCVS. However, the underlying molecular mechanisms are yet to be elucidated, therefore, further studies are required in the future.

\section{References}

1. Wencel K, Dyaczyńska-Herman A and Wencel T: Plasma clotting and fibrinolysis parameters in patients with spontaneous and posttraumatic SAH. Clin Neuro Neurosurg 99 (Suppl 1): 70-70, 1997.

2. Arreche ND, Sarati LI, Martinez CR, Fellet AL and Balaszczuk AM: Contribution of caveolin-1 to ventricular nitric oxide in age-related adaptation to hypovolemic state. Regul Pept 179: 43-49, 2012.

3. Agyemang C, van Oeffelen AA, Norredam M, et al: Ethnic disparities in ischemic stroke, intracerebral hemorrhage, and subarachnoid hemorrhage incidence in The Netherlands. Stroke 45: 3236-3242, 2014

4. Laskowitz DT and Kolls BJ: Neuroprotection in subarachnoid hemorrhage. Stroke 41 (Suppl 10): S79-S84, 2010.

5. Connolly ES Jr, Rabinstein AA, Carhuapoma JR, Derdeyn CP, Dion J, Higashida RT, Hoh BL, Kirkness CJ, Naidech AM, Ogilvy CS, et al: Guidelines for the management of aneurysmal subarachnoid hemorrhage: A guideline for healthcare professionals from the American heart association/American stroke association. Stroke 43: 1711-1737, 2012.

6. Pluta RM (ed): Introduction to problems of postsubarachnoid hemorrhage delayed cerebral vasospasm. In: Animal Models of Acute Neurological Injuries II. Humana Press, New York, NY, 459-464, 2012

7. Anderson GB, Ashforth R, Steinke DE, et al: CT angiography for the detection of cerebral vasospasm in patients with acute subarachnoid hemorrhage. Am J Neuroradiol 21: 1011-1015, 2000.

8. Specogna AV: Subarachnoid hemorrhage diagnosis. JAMA 311: 201,2014

9. Huang W and Zhou Z: Research advances on pathogenesy of cerebral vasospasm after suharachnoid hemorrhage. Zhong Guo Nao Xue Guan Bing Za Zhi 7: 215-219, 2010 (In Chinese).

10. Roos YB, Dijkgraaf MG, Albrecht KW, Beenen LF, Groen RJ, de Haan RJ and Vermeulen M: Direct costs of modern treatment of aneurysmal subarachnoid hemorrhage in the first year after diagnosis. Stroke 33: 1595-1599, 2002.

11. Pluta RM: Delayed cerebral vasospasm and nitric oxide: Review, new hypothesis, and proposed treatment. Pharmacol Ther 05: 23-56, 2005.

12. Suzuki H, Kanamaru K, Tsunoda H, Inada H, Kuroki M, Sun H, Waga S and Tanaka T: Heme oxygenase-1 gene induction as an intrinsic regulation against delayed cerebral vasospasm in rats. J Clin Invest 104: 59-66, 1999.

13. Varsos VG, Liszczak TM, Han DH, Kistler JP, Vielma J, Black PM, Heros RC and Zervas NT: Delayed cerebral vasospasm is not reversible by aminophylline, nifedipine, or papaverine in a 'two-hemorrhage' canine model. J Neurosurg 58: 11-17, 1983.

14. Manabe K, Ito H, Matsuda $\mathrm{H}$, et al: Hyperpolarization induced by vasoactive substances in intact guinea-pig endocardial endothelial cells. J Physiol 484: 25-40, 1995.

15. Rabinstein AA, Lanzino G and Wijdicks EF: Multidisciplinary management and emerging therapeutic strategies in aneurysmal subarachnoid haemorrhage. Lancet Neurol 9: 504-519, 2010.

16. Rothberg KG, Heuser JE, Donzell WC, Ying YS, Glenney JR and Anderson RG: Caveolin, a protein component of caveolae membrane coats. Cell 68: 673-82, 1992.

17. Zhang $\mathrm{H}$ and Zou W: Caveolin-1 and Breast Cancer. Chinese Zhong Guo Sheng Wu Hua Xue Yu Fen Zi Sheng Wu Xue Bao 23: 20-26, 2007 (In Chinese).

18. Sampson MJ, Lovell RS and Craigen WJ: The murine voltage-dependent anion channel gene family. Conserved structure and function. J Biol Chem 272: 18966-18973, 1997.

19. Hurlstone AF, Reid G, Reeves JR, Fraser J, Strathdee G, Rahilly M, Parkinson EK and Black DM: Analysis of the CAVEOLIN-1 gene at human chromosome $7 \mathrm{q} 31.1$ in primary tumours and tumour-derived cell lines. Oncogene 18: 1881-1890, 1999. 
20. Prinetti A, Prioni S, Loberto N, Aureli M, Chigorno V and Sonnino S: Regulation of tumor phenotypes by caveolin-1 and sphingolipid-controlled membrane signaling complexes. Biochim Biophys Acta 1780: 585-596, 2008.

21. Botos E, Klumperman J, Oorschot V, Igyártó B, Magyar A, Oláh $\mathrm{M}$ and Kiss AL: Caveolin-1 is transported to multi-vesicular bodies after albumin-induced endocytosis of caveolae in HepG2 cells. J Cell Mol Med 12: 1632-1639, 2008.

22. Arakawa R, Abedohmae S, Asai M, Ito JI and Yokoyama S: Involvement of caveolin-1 in cholesterol enrichment of high density lipoprotein during its assembly by apolipoprotein and THP-1 cells. J Lipid Res 41: 1952-1962, 2001.

23. Yu Q, Chen X, Fang X, Chen Q and Hu C: Caveolin-1 aggravates cigarette smoke extract-induced MUC5AC secretion in human airway epithelial cells. Int J Mol Med 35: 1435-1442, 2015.

24. SmartEJ, Graf GA, McNiven MA, Sessa WC, Engelman JA, Scherer PE, Okamoto T, Lisanti MP: Caveolins, liquid-ordered domains, and signal transduction. Mol Cell Biol 19: 7289-7304, 1999.

25. Song L, Liu J, Zou W and An LJ: Role of Caveolin-1 in cell senescence and senescent dieases. Zhong Guo Sheng Wu Hua Xue Yu Fen Zi Sheng Wu Xue Bao 08: 712-720, 2011 (In Chinese).

26. Jasmin JF, Malhotra S, Singh Dhallu M, Mercier I, Rosenbaum DM and Lisanti MP: Caveolin-1 deficiency increases cerebral ischemic injury. Circ Res 100: 721-729, 2007

27. Shen J, Ma S, Chan P, Lee W, Fung PC, Cheung RT, Tong Y and Liu KJ: Nitric and oxide down-regulates caveolin-1 expression in rat brains during focal cerebral ischemia and reperfusion injury. J Neurochem 96: 1078-1089, 2006.

28. Lee JY, Huang DL, Keep R and Sagher O: Characterization of an improved double hemorrhage rat model for the study of delayed cerebral vasospasm. J Neurosci Methods 168: 358-366, 2008.

29. Bederson JB, Pitts LH, Tsuji M, Nishimura MC, Davis RL and Bartkowski H: Rat middle cerebral artery occlusion: Evaluation of the model and development of a neurologic examination. Stroke 17: 472-476, 1986

30. Vellimana AK, Milner E, Azad TD, Harries MD, Zhou ML, Gidday JM, Han BH and Zipfel GJ: Endothelial nitric oxide synthase mediates endogenous protection against subarachnoid hemorrhage-induced cerebral vasospasm. Stroke 42: 776-782, 2011

31. Kassell NF, Sasaki T, Colohan AR and Nazar G: Cerebral vasospasm following aneurysmal subarachnoid hemorrhage. Stroke 1985, 16 $562-572,1985$.

32. Jia L and Sun BL: Establishment of an animal model of cerebral vasospasm following subarachnoid hemorrhage. J Clinical Rehabilitative Tissue Engineering Research 13: 8147-8150, 2009.

33. Sugawara T, Ayer R, Jadhav V and Zhang JH: A new grading system evaluating bleeding scale in filament perforation subarachnoid hemorrhage rat model. J Neurosci Methods 167: 327-334, 2008

34. Marbacher S, Fandino J and Kitchen ND: Standard intracranial in vivo animal models of delayed cerebral vasospasm. Br J Neurosurg 24: 415-434, 2010.
35. Güresir E, Raabe A, Jaiimsin A, Dias S, Raab P, Seifert V and Vatter H: Histological evidence of delayed ischemic brain tissue damage in the rat double-hemorrhage model. J Neurol Sci 293: 18-22, 2010.

36. Yanamoto H, Kataoka H, Nakajo $\mathrm{Y}$ and Iihara K: The role of the host defense system in the development of cerebral vasospasm: Analogies between atherosclerosis and subarachnoid hemorrhage. Eur Neurol 68: 329-343, 2012.

37. Chaichana KL, Pradilla G, Huang J and Tamargo RJ: Role of inflammation (leukocyte-endothelial cell interactions) in vasospasm after subarachnoid hemorrhage. World Neurosurg 73: 22-41, 2010.

38. Anuntasethakul T, Srikiatkhachorn A, Maneesri S, Patumraj S and Kasantikul V: Ultrastructural changes in endothelial cells of cerebral microvessels after exposure to nitric oxide donor. Neuropathol 19: 259-266, 1999.

39. Pluta RM, Andre D, George G, Gladwin MT and Oldfield EH: Nitrite infusions to prevent delayed cerebral vasospasm in a primate model of subarachnoid hemorrhage. JAMA 293: $1477-1484,2005$.

40. Kevil CG and Patel RP: Preserving vessel function during ischemic disease: New possibilities of inorganic nitrite therapy. Expert Rev Cardiovasc Ther 6: 1175-1179, 2008.

41. Duncan C, Dougall H, Johnston P, Green S, Brogan R, Leifert C, Smith L, Golden M and Benjamin N: Chemical generation of nitric oxide in the mouth from the enterosalivary circulation of dietary nitrate. Nat Med 1: 546-551, 1995.

42. Dora KA, Doyle MP and Duling BR: Elevation of intracellular calcium in smooth muscle causes endothelial cell generation of NO in arterioles. PNAS 94: 6529-6534, 1997.

43. Corpas FJ, Barroso JB, Carreras A, Quirós M, León AM, Romero-Puertas MC, Esteban FJ, Valderrama R, Palma JM, Sandalio LM, et al: Cellular and subcellular localization of endogenous nitric oxide in young and senescent pea plants. Plant Physiol 136: 2722-2733, 2004.

44. Russell FD and Molenaar P: The human heart endothelin system: ET-1 synthesis, storage, release and effect: Trends Pharmacol Sci 21: 353-359, 2000

45. Thyberg J: Caveolin-1 and caveolae act as regulators of mitogenic signaling in vascular smooth muscle cells. Arterioscler Thrombo Vasc Biol 23: 1481-1483, 2003.

46. Penumathsa SV, Koneru S, Samuel SM, Maulik G, Bagchi D, Yet SF, Menon VP and Maulik N: Strategic targets to induce neovascularization by resveratrol in hypercholesterolemic rat myocardium: role of caveolin-1, eNOS, $\mathrm{HO}-1$ and VEGF. Free Radic Biol Med 45: 1027-1034, 2008.

47. Zhang X, Zhou Z, Liu J, Feng B, Zhang J and Huang W: Relationship between caveolin-1 and proliferation of basilar artery smooth muscle in rabbit model of subarachnoid hemorrhage. Journal of Third Military Medical University 33: 830-834, 2011 